This is the accepted version

Live link to published version in IEEE Xplore: https://ieeexplore.ieee.org/document/8561163

Citation to the original IEEE publication: A. Thakallapelli, S. Ghosh, and S. Kamalasadan, "Development and Applicability of Online Passivity Enforced Wide-Band Multi-Port Equivalents For Hybrid Transient Simulation”, IEEE Trans. on Power Syst., Volume: 34, No: 3, pp. 2302 - 2311 , May. 2019.

Digital Object Identifier (DOI): 10.1109/TPWRS.2018.2885240

The following copyright notice is displayed here as per Operations Manual Section 8.1.9 on Electronic Information Dissemination (known familiarly as "author posting policy"):

(C) 2018 IEEE. Personal use of this material is permitted. Permission from IEEE must be obtained for all other uses, in any current or future media, including reprinting/republishing this material for advertising or promotional purposes, creating new collective works, for resale or redistribution to servers or lists, or reuse of any copyrighted component of this work in other works. 


\title{
Development and Applicability of Online Passivity Enforced Wide-Band Multi-Port Equivalents For Hybrid Transient Simulation
}

\author{
Abilash Thakallapelli, Student Member, IEEE, Sudipta Ghosh, Member, IEEE, \\ and Sukumar Kamalasadan, Senior Member, IEEE
}

\begin{abstract}
This paper presents a method for developing single and multi-port Frequency Dependent Network Equivalent (FDNE) based on a passivity enforced online recursive least squares (RLS) identification algorithm which identifies the input admittance matrix in $z$-domain. Further, with the proposed architecture, a real-time hybrid model of the reduced power system is developed that integrate Transient Stability Analysis (TSA) and FDNE. Main advantages of the proposed architecture are, it identifies the FDNE even with unknown network parameters in the frequency range of interest, and yet can be implemented directly due to discrete formulation while maintaining desired accuracy, stability and passivity conditions. The accuracy and characteristics of the proposed method are verified by implementing on two-area, IEEE 39 and 68 bus power system models.
\end{abstract}

Index Terms-Electromagnetic Transient (EMT) Simulation, Transient Stability Analysis, Frequency Dependent Network Equivalent, Recursive Least Square Identification (RLS), Aggregated Generator Model (AGG).

\section{NOMENCLATURE}

EMT Model

EMT+TSA

$\mathrm{EMT}+\mathrm{TSA}(\mathrm{AGG})$

$\mathrm{EMT}+\mathrm{FDNE}$

$\mathrm{EMT}+\mathrm{FDNE}(\mathrm{VF})$

EMT+FDNE+TSA

EMT+FDNE+TSA (AGG) The external area is modeled as a combination of FDNE and TSA type with both network and generator aggregation.

A. Thakallapelli, S. Ghosh, and S. Kamalasadan (corresponding author), are with the Power, Energy and Intelligent Systems Laboratory, Energy Production Infrastructure Center (EPIC) and Department of Electrical Engineering, University of North Carolina at Charlotte, Charlotte, NC 28223 USA (e-mail: athakall@uncc.edu,sghosh9@uncc.edu,skamalas@uncc.edu).

\section{INTRODUCTION}

$\mathbf{R}$ EAL-TIME EMT simulation requires detailed modeling of transmission systems to understand the effect of transients and harmonics arising due to varying operating conditions and disturbances in power grid. Effect of power electronic components associated with renewable energy sources on the power grid and performance of different controllers can be analyzed using EMT simulations [1]. Typically, integration time step of EMT simulation is in microseconds $(\mu s)$. This makes modeling of a large transmission system for EMT studies impractical as detail modeling increases complexity and computational burden. One solution is to model the transmission system as TSA type with larger integration time as TSA simulations can run faster than EMT. However, in TSA type, due to large integration time, high-frequency transients following a disturbance in the system are not preserved making this approach not very accurate [2].

Another approach is to model large transmission network as frequency dependent reduced order systems that can represent the power grid under any operating condition. One way to reduce large power grid is to model part of the grid which is of interest (study area) in detail and the remainder of the system (external area) by an efficient equivalent such as FDNE [3]. In this process, initially, the network is divided into study and external area based on the coherency grouping of generators such that all coherent generators are present in the external area [4]. The boundary between the study and external area is divided considering the fact that interconnecting points should have the least minimum number of ports. Generally, in TSA type equivalent, the network admittance is evaluated only at the fundamental frequency, hence this representation ignores high-frequency oscillations. The high-frequency behavior of the external area can be preserved by using FDNE [5], however, FDNE ignores electromechanical oscillations. In order to cover both electromechanical and high-frequency behavior, the external area should be modeled as a combination of TSA equivalent and FDNE.

FDNEs are generally formulated as frequency-dependent black-box terminal equivalents based on rational functions. In [6], FDNE is formulated using modal vector fitting (VF) method and in [7] passivity of the rational function is enforced using mode revealing algorithm. The authors in [8], used VF method to generate FDNE combined with TSA component for real-time simulation and, in [9] this method was improved 
by including generator coherency. Further, the applicability of these models for large power system real-time simulation is studied in [10]. Formulation of FDNE using time domain VF and simulated time domain response evaluations are performed in [3], and in [11], authors compared different rational approximation methods for simulated time domain responses. A rational approximation approach for formulating FDNE based on matrix pencil method is proposed in [12] where as [13] proposed a method for electromechanical and electromagnetic transient analysis using FDNE.

However, state-of-the-art algorithms formulate FDNE with continuous domain transfer function which makes it very complex to implement these algorithms in the real-time simulator (EMT based). Also, in many of these methods FDNE formulation is dependent on the availability of admittance data over a wide range of frequency which is not always possible. Further, off-line calculation of admittance over a wide frequency range, and storing and retrieving of the data for curve fitting is tedious and time consuming. For example, if the frequency range of interest is from 0 to $5000 \mathrm{~Hz}$ with a step size of $0.1 \mathrm{~Hz}$, one should construct 50001 admittance matrices at each frequency sample which is extremely complex to compute. This complexity increases with sample size, number of ports, and size of network under consideration.

This paper introduces a novel method for formulating FDNEs based on online RLS identification. In this method, the external area is energized with constant voltage source at the boundary buses after all the voltage sources and current sources in the network are short-circuited and open circuited respectively. Subsequently, by tracking input voltage and output current, FDNE is formulated. The proposed method simplifies FDNE formulation as it is independent of the availability of network parameters over a wide frequency range and its formulation is in discrete domain directly. Also the computational burden is reduced by using Kron's node elimination method in the external area network. The advantages of the proposed architecture are:

- FDNE formulation is independent of the availability of network parameters over a wide frequency range.

- The architecture formulates FDNE in discrete domain, which reduces complexity in interfacing FDNE with the real-time simulator.

- The architecture formulates coherency based network equivalents of complex networks with less computational burden and desired accuracy.

- The methodology enforces stability and passivity conditions to ensure stable EMT simulations.

- The methodology can be directly implemented for realtime control [14].

The rest of the paper is organized as follows: In section II overall architecture is discussed. In section III implementation test on the interconnected power grid is discussed and Section IV concludes the paper.

\section{Proposed Methodology}

In large power grids, it has been frequently observed that, after disturbances, the generators swing together in groups, meaning units near a disturbance respond faster and together, whereas distant machines show relatively damped oscillatory behavior. This physical property is known as coherency and group of machines with similar responses are termed coherent generators. In our work, the generators are coherently grouped based on a localness index. Further, power system model order reduction is performed by dividing the original system into a study area and an external area. The proposed method further divides the external area into two parts. The first one is a low-frequency equivalent (TSA) and the second one is highfrequency equivalent (FDNE). The reduced order modeling of power system involves the following steps:

\section{A. Aggregation of External Area for TSA type modeling and Real-time Integration}

For retaining the electromechanical behavior of the system under consideration, aggregated TSA model is used. Fig. 1 shows the flowchart for TSA type modeling.

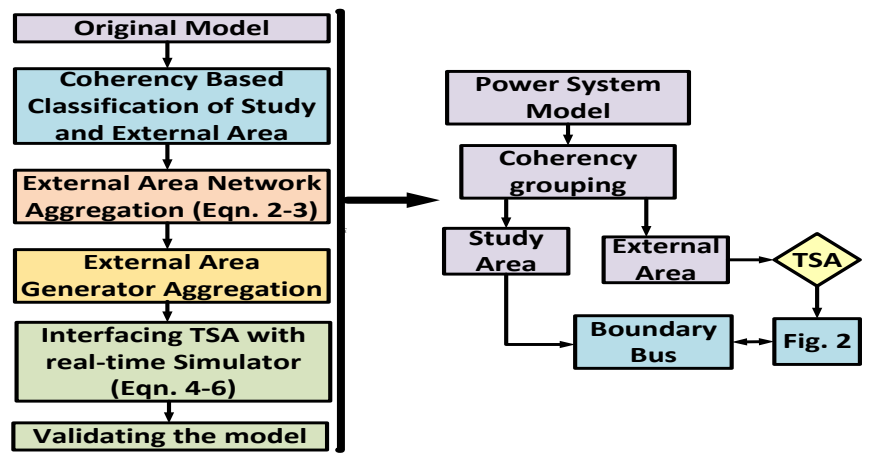

Fig. 1. The conceptual and functional flowchart for TSA type modeling

1) Coherency Based Classification of Study and External Area: For coherency grouping of the power system under study, initially the small signal stability study of the power grid model is performed and the generator electromechanical modes of oscillation are evaluated. Further, based on participation factors of all the generators, a localness index is calculated as follows:

$$
L_{\text {index }, i}=\sum_{k=1}^{n}\left(1-P_{k i}\right)^{n}
$$

where $n$ is a number of synchronous generators connected in the system and, $P_{k i}$ is the normalized participation factor of the $k^{\text {th }}$ machine in the $i^{\text {th }}$ mode. For example, Table. I shows the coherency grouping of generators for IEEE 39 bus system based on the localness index. More details of the localness index are discussed in [15].

TABLE I

COHERENCY GROUPING OF GENERATORS FOR IEEE 39 BUS SYSTEM

\begin{tabular}{ccccc}
\hline Group & I & II & III & IV \\
\hline Generators & $4,5,6,7,9$ & $1,10,8$ & 3 & 2 \\
\hline
\end{tabular}

2) Network Aggregation: For aggregating the external area network, the admittance matrix $\left(Y_{n \times n}\right)$ of the external area is formulated using the bus and line data (at $60 \mathrm{~Hz}$ ). For example, if there are $n$ number of buses in the external area and we want to retain $m$ buses (i.e. $m=i+j$, where $j$ be the number of boundary buses and $i$ is the number of generator buses) and 
eliminate the remaining $n-m$ buses in the external area, then using Kron node elimination method [16], reduced admittance matrix $\left(Y_{\text {red }}\right)$ can be obtained as:

$$
\begin{aligned}
& Y_{r e d(m \times m)}=\left[Y_{m \times m}-Y_{m \times n} Y_{n \times n}^{-1} Y_{n \times m}\right] \\
& {\left[\begin{array}{c}
I_{b(j \times 1)} \\
I_{g(i \times 1)}
\end{array}\right]=Y_{r e d(m \times m)}\left[\begin{array}{c}
V_{b(j \times 1)} \\
V_{g(i \times 1)}
\end{array}\right]}
\end{aligned}
$$

where subscript $b$ and $g$ represents the boundary and generator buses respectively.

3) Generator and Associated Controller Aggregation: After network aggregation and generators are left intact, the reduced admittance matrix is of the size $m \times m(m=i+j$, i.e. there are $j$ boundary buses and $i$ generator buses). To further reduce computational burden and to reduce complexity in modeling, generators and associated controllers can be aggregated. With generator aggregation, the reduced admittance matrix is of the size $(m-i+1) \times(m-i+1)(m=i+j$, i.e. $i$ coherent generators can be aggregated into one generator). In the case where generators are not aggregated the accuracy of coherency grouping has no significant effect on the TSA, but in the case of generator aggregation, all the generators which are to be aggregated must be in the same coherent group. For scenarios where multiple operating condition changes, the grouping can be performed using online coherency grouping [17]. Method of generator and controller aggregation is discussed in [18]. Thus additional details are not explained in the paper.

4) Interfacing TSA type modeling with real-time simulator: In this step, voltages at the boundary buses are the input to the TSA block, where output currents from the TSA block are injected back to the boundary buses. Here, the generators are modeled in detail to observe the electromechanical behavior. Conversion of boundary bus voltage from time domain to phasor domain is then performed using discrete sequence analyzer [19]. The result gives magnitude $\left|V_{b}\right|$ and, phase angle $\angle V_{b}$. Also, phase angle of $V_{b}$ with reference to $I_{b}$ can be determined as $\theta_{b}=\angle \delta_{b}-\angle V_{b}$, where $\angle \delta_{b}$ is the angle of $I_{b}$. Then, using (4) the generator bus voltage can be calculated as

$$
V_{g} \angle \theta_{g}=\left(I_{g} \angle \delta_{g}-Y_{g b} V_{b} \angle \theta_{b}\right) Y_{g g}^{-1}
$$

where $I_{g}$ is the generator current injection, $\angle \delta_{g}$ is the angle of generator current, $V_{b}$ is the boundary bus voltage, $V_{g}$ is the generator bus voltage and, $\angle \theta_{g}$ is the angle of generator bus voltage. Using $I_{g}$ (for the first iteration the initial value is obtained from the power flow solution) and $V_{b}$, the generator voltage $V_{g}$ is calculated. The generator bus is energized with $V_{g}$ and subsequently $I_{g}$ is obtained from generator phasor model. From calculated $V_{g}$ and boundary bus voltage $V_{b}$, boundary bus current injection $I_{i n j}$ is calculated as shown in (5)-(6). A graphical description is shown in Fig. 2.

$$
\begin{gathered}
I_{b} \angle \delta_{b}=Y_{b b} V_{b} \angle \theta_{b}+Y_{b g} V_{g} \angle \theta_{g} \\
I_{i n j} \angle \beta_{b}=I_{b} \angle \delta_{b}
\end{gathered}
$$

After calculating boundary bus current $I_{i n j} \angle \beta_{b}$ in phasor form, it is converted into the time domain and injected into the boundary bus. The overall implementation is shown in Fig. 1.

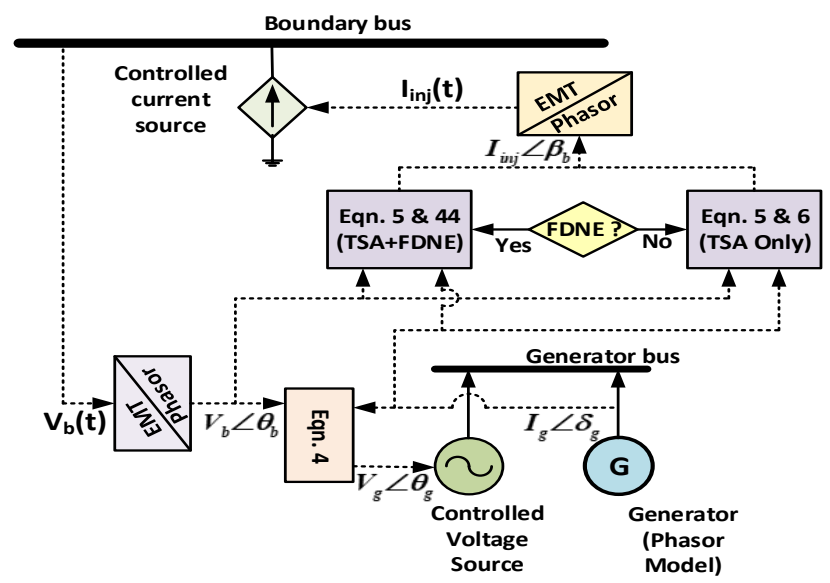

Fig. 2. TSA Calculation

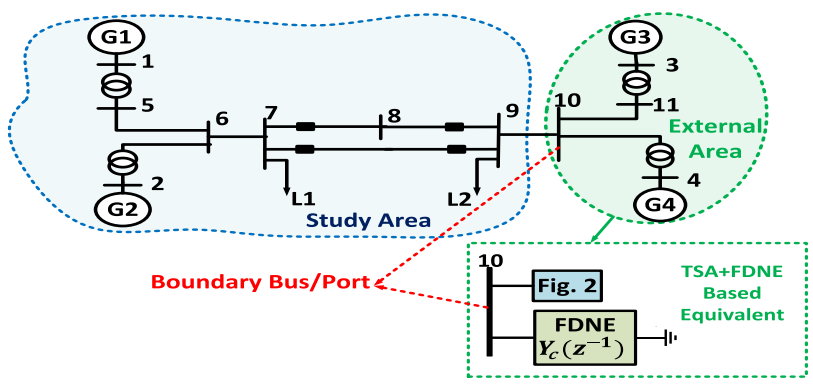

Fig. 3. Proposed dynamic equivalent of two area system

\section{B. Study and analysis of TSA type equivalent modeling}

For study and preliminary analysis, the TSA type equivalent is implemented on two area power system model as shown in Fig. 3. In this test system, area-1 consists of generators G1, G2, and area- 2 consists of generators G3, G4 [20]. For analysis purpose, area- 2 is considered as external area with boundary bus as bus 10 . Two cases are considered here. In the first case EMT+TSA Based Model and in the second case EMT+TSA Based Model $(A G G)$ are analyzed. For bench-marking, both the test cases are compared with (EMT Based Model).

For validating the proposed approach a 3-ph fault is created on Bus- 8 at $1 \mathrm{~s}$ for a duration of $0.1 \mathrm{~s}$. Fig. 4 shows the comparison of the relative speed of Gen. 2 with respect to (w.r.t) gen. 1, and Fig. 5 shows the comparison of active power flow from bus 10 to bus 9. In general, after a disturbance, highfrequency transients occur for a short duration whereas electromechanical oscillation will be for longer duration. From Fig. 4, it can be observed that due to large simulation time step compared to EMT type, TSA type equivalent can only preserve electro-mechanical oscillations, but high-frequency oscillations are not preserved as it can be seen from Fig. 5.

For quantitative analysis, relative error between the two cases and full EMT type model is calculated using (7). The results are tabulated in Table. II.

$$
\text { relative error }=\frac{\left\|y_{\text {ref }}-y_{\text {act }}\right\|_{2}}{\left\|y_{\text {ref }}\right\|_{2}}
$$

where $y_{\text {ref }}$ represents the output from full EMT model (EMT Based Model) and $y_{\text {act }}$ represents the output obtained in each 


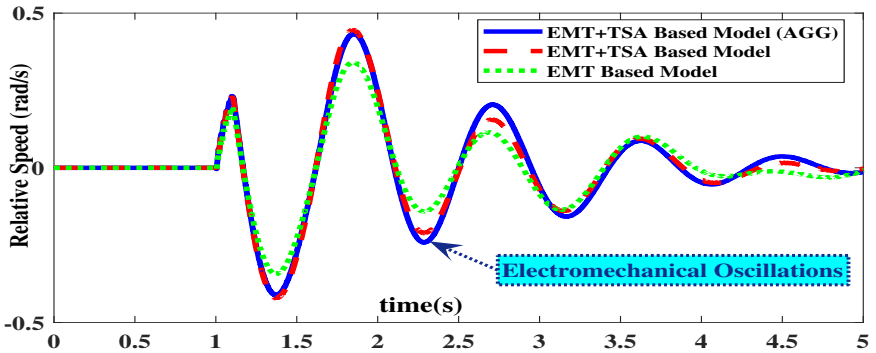

Fig. 4. The relative speed of Gen.2 w.r.t Gen.1

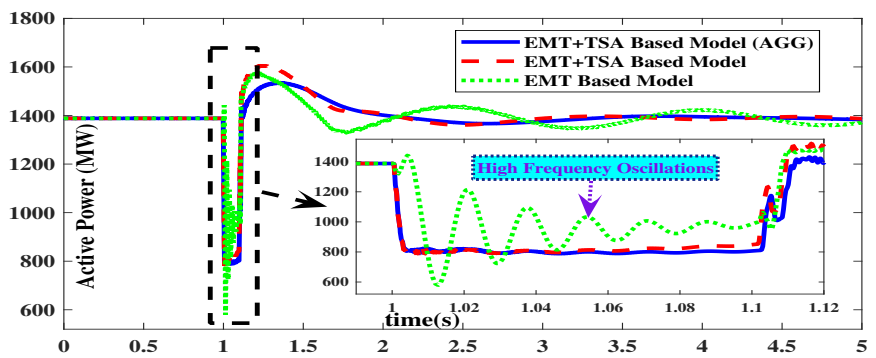

Fig. 5. Active power flow from bus 10 to bus 9

case (EMT+TSA Based Model (AGG) and (EMT+TSA Based Model) respectively)

TABLE II

COMPARISON OF REDUCED (EMT+TSA) AND ORIGINAL (EMT) MODELS

\begin{tabular}{ccc}
\hline & EMT+TSA(AGG) & EMT+TSA \\
\hline Relative Speed (Fig. 4) & 0.3589 & 0.2998 \\
\hline Active Power (Fig. 5) & 0.0356 & 0.0351 \\
\hline
\end{tabular}

It can be seen that the aggregated model has a large error. This motivates the use of FDNE representations.

\section{FDNE Formulation and Real-time simulator Integration}

For retaining the high-frequency behavior of the system under consideration, FDNE is formulated. Fig. 6 shows the FDNE modeling flowchart. In this method, the external area is energized with a constant voltage source with varying frequency in steps of 0.01 (from few $\mathrm{Hz}$ to $\mathrm{kHz}$ ) after shortcircuiting all voltage sources, and open circuiting all current sources. Since, the goal here is to identify the frequency dependent admittance $\left(Y_{f}\right)$, the boundary bus voltage and current are the required signals. FDNE is then formulated in $z$-domain using RLS [21], [22] by tracking input voltage and output current. The basic principle is as follows. If $V_{F}$ is the voltage input to the boundary bus and $I_{F}$ is the current output from the boundary bus, then $Y_{f}$ can be written as

$$
Y_{f}\left(z^{-1}\right)=\frac{I_{F}(k)}{V_{F}(k)}=\frac{b_{1} z^{-1}+b_{2} z^{-2}+\ldots+b_{n} z^{-n}}{1+a_{1} z^{-1}+a_{2} z^{-2}+\ldots+a_{n} z^{-n}}
$$

where $k$ is the number of samples. For a $m$-port network (that means $m$ boundary buses), $Y_{f}$ can be represented as in (9), where $Y_{f(m, m)}$ and $Y_{f(m, p)}$ in (9) are the self and mutual admittance respectively. FDNE model formulation and validation involves the following steps.

1) Recursive Least Square Estimation: Identification of a dynamic process is performed using the process input $u(k)$ and

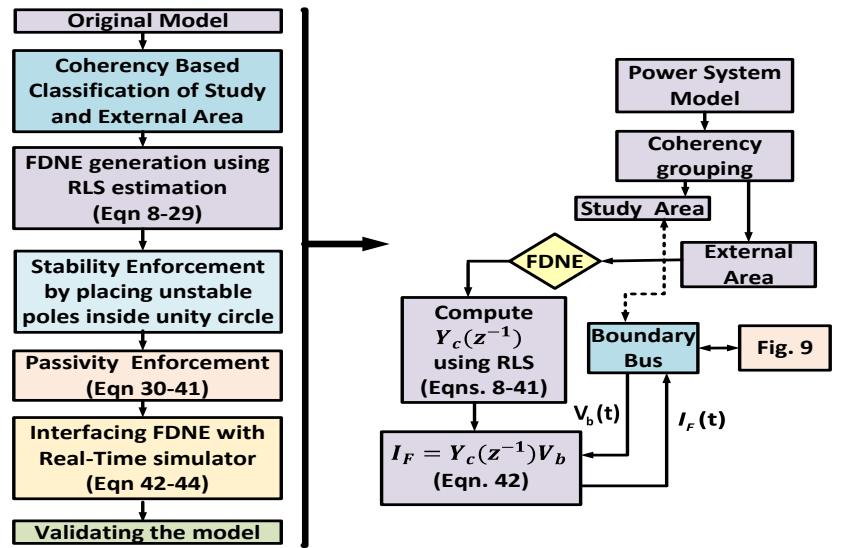

Fig. 6. The conceptual and functional flowchart for FDNE modeling the process output $y(k)$ at every sample $k$. Considering the $z$ domain model of an $n^{t h}$ order process, this can be represented as

$$
\frac{y(k)}{u(k)}=\frac{b_{1} z^{-1}+b_{2} z^{-2}+\cdots+b_{n} z^{-n}}{1+a_{1} z^{-1}+a_{2} z^{-2}+\cdots+a_{n} z^{-n}}
$$

where $a^{\prime} s$ and $b^{\prime} s$ are the transfer function denominator and numerator coefficients respectively. For $N$ observation window length, (10) can be rewritten as

$$
\left[\begin{array}{c}
y(k) \\
y(k-1) \\
\cdot \\
\cdot \\
\cdot \\
y(k-N+1)
\end{array}\right]_{N \times 1}=\left[X_{N \times 2 n}\right]\left[\begin{array}{c}
a_{1} \\
\cdot \\
\cdot \\
a_{n} \\
b_{1} \\
\cdot \\
\cdot \\
b_{n}
\end{array}\right]_{2 n \times 1}
$$

Equation (11) can be written in the generic form as

$$
\Phi_{\text {model }(N \times 1)}=X_{N \times 2 n} \Theta_{2 n \times 1}
$$

where $X$ is a matrix of past inputs and outputs, $\Phi$ is a matrix of past and present outputs, and $\Theta$ is the coefficient matrix of the transfer function.

Assume that the model identified is different from measurements, then

$$
\epsilon=\Phi_{\text {measured }}-\Phi_{\text {model }}
$$

where $\epsilon$ is the error between the performance of the system measurement (subscript measured) and the model (subscript model). For reducing this error, a criteria $J$ can be defined as

$$
J=\epsilon^{t} \epsilon
$$

By letting $d J / d \Theta=0$, we get

$$
\Theta=\left[X^{t} X\right]^{-1} X^{t} \Phi_{\text {measured }}
$$

From (15) it can be seen that, in order to calculate the measured variable the inverse of the state matrix should be determined. This can drastically slow down the process and some time may not be achievable. To circumvent this issue, an RLS based computational algorithm that eliminates the matrix inversion is designed. Let $S=X^{t} X$, then (15) can be written as

$$
\Theta=S^{-1} X^{t} \Phi
$$




$$
Y_{f}\left(z^{-1}\right)_{m \times m}=\left[\begin{array}{cccc}
Y_{f(1, p)}+Y_{f(1,1)}+\cdots+Y_{f(1, m)} & -Y_{f(1,2)} & \ldots & -Y_{f(1, m)} \\
Y_{f(2,1)} & \cdot & \ldots & -Y_{f(2, m)} \\
\cdot & \cdot & \ldots & \cdot \\
-Y_{f(m, 1)} & \cdot & \ldots & Y_{f(m, p)}+Y_{f(m, 1)}+\cdots+Y_{f(m, m)}
\end{array}\right]
$$

where $\Phi=\Phi_{\text {measured }}$ Then,

$$
\begin{gathered}
\Theta(k)=S^{-1}\left[X(k) X^{t}(k-1)\right]\left[\begin{array}{c}
\Phi(k) \\
\Phi(k-1)
\end{array}\right] \\
\Theta(k)=S^{-1}\left[X(k) \Phi(k)+X^{t}(k-1) \Phi(k-1)\right]
\end{gathered}
$$

Using (12), (18) can be written as

$$
\begin{gathered}
\Theta(k)=S^{-1}\left[X(k) \Phi(k)+X^{t}(k-1) X(k-1) \Theta(k-1)\right] \\
\Theta(k)=S^{-1}[X(k) \Phi(k)+S(k-1) \Theta(k-1)] \\
S(k)=S(k-1)+X(k) X^{t}(k)
\end{gathered}
$$

Substituting (21) in (20)

$$
\begin{array}{r}
\Theta(k)=S^{-1}\left[X(k) \Phi(k)+\left\{S(k)-X(k) X^{t}(k)\right\} \Theta(k-1)\right] \\
\Theta(k)=\Theta(k-1)+\left[S(k-1)+X(k) X^{t}(k)\right]^{-1} X(k) \\
{\left[\Phi(k)-X^{t}(k) \Theta(k-1)\right]}
\end{array}
$$

Let $P(k)=S^{-1}(k)$. Then by using matrix inversion lemma, $P(k)$ can be represented as

$$
P(k)=P(k-1)\left[I-\frac{X(k) X^{t}(k)}{1+X^{t}(k) P(k-1) X(k)}\right] P(k-1)
$$

Let

$$
K(k)=\frac{X(k)}{1+X^{t}(k) P(k-1) X(k)}
$$

Then, $P(k)$ can be re-written as

$$
P(k)=\left[I-K(k) X^{t}(k)\right] P(k-1)
$$

Substituting (26) in (23), (23) can be represented as

$$
\Theta(k)=\Theta(k-1)+K(k)\left[\Phi(k)-X^{t}(k) \Theta(k-1)\right]
$$

With weighted least square, (25) and (26) can be presented as

$$
\begin{gathered}
K(k)=\frac{P(k-1) X(k)}{\gamma+X^{t}(k) P(k-1) X(k)} \\
P(k)=\frac{\left[I-K(k) X^{t}(k)\right] P(k-1)}{\gamma}
\end{gathered}
$$

where $\gamma$ is the weighting factor.

Thus, with a given process input $V_{F}(k)$ and process output $I_{F}(k), Y_{f}$ can be computed using RLS estimation. The validity of the proposed algorithm is verified by implementing on different test systems. In the first case, the proposed algorithm is implemented on two area test system with 1-port network. Fig. 7(a) and Fig. 7(b) shows the magnitude and angle of admittance of the external area and Table. III shows the comparison of FDNE formulation for two area system. It can be observed that even though this approach uses lower order transfer function (meaning less computational burden), it gives similar error compared to higher order VF method.

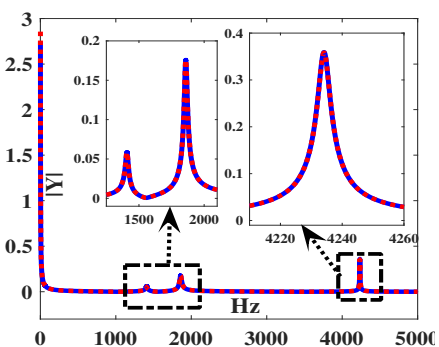

(a) Magnitude of admittance

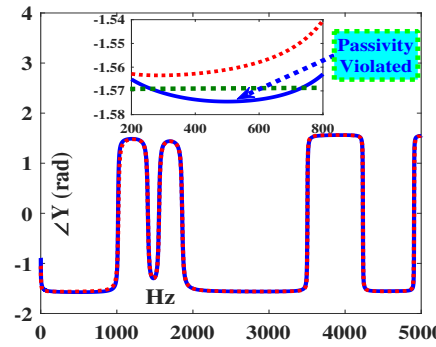

(b) Angle of admittance

$$
\text { - Proposed *..... Original Response }
$$

Fig. 7. Admittance vs frequency of external area (two area system)

TABLE III

FDNE FORMULATION COMPARISON

\begin{tabular}{ccc}
\hline Type & Proposed Method & Vector Fitting(VF) \\
\hline FDNE Order & 17 & 21 \\
\hline RMS Error & $2.176 \mathrm{e}-6$ & $2.0101 \mathrm{e}-6$ \\
\hline
\end{tabular}

2) Passivity Enforcement: For a stable EMT simulation, the admittance matrix should be passive. Presence of negative resistance (i.e angle of admittance $> \pm 1.57 \mathrm{rad}$ in phasor form) in the frequency range of interest due to inherent approximations in identification violates passivity. For instance, from Fig. 7(b) it can be seen that for the previous test case, passivity is violated. For enforcing passivity, an algorithm is developed as shown in Algorithm 1. The details are as follows.

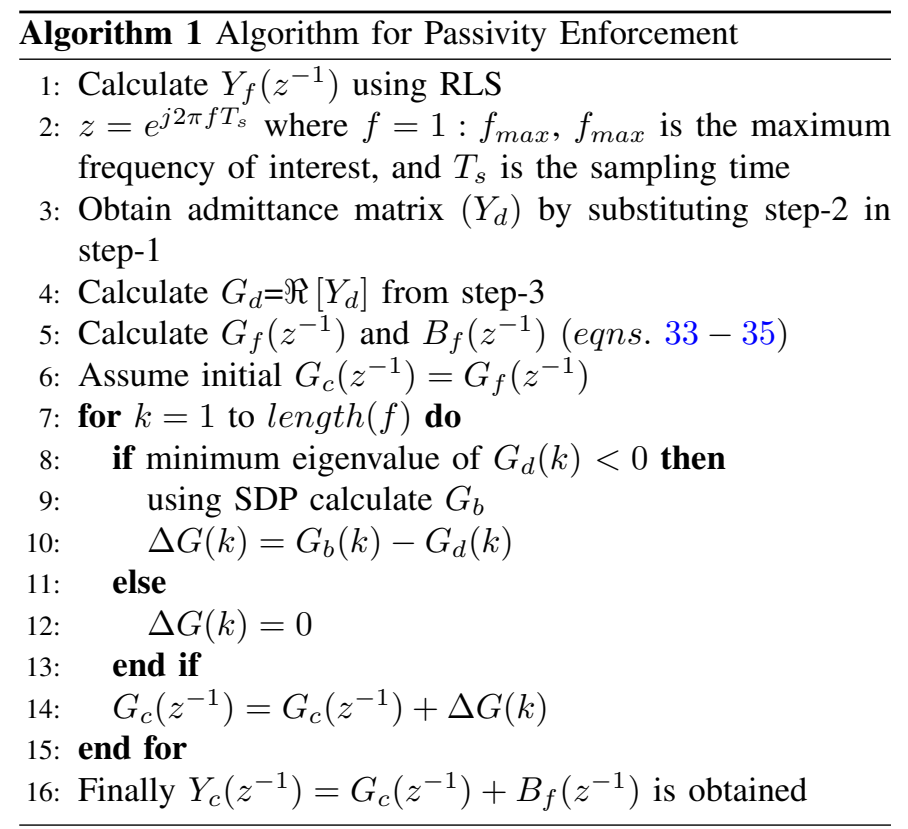

From (9), let $Y_{f}\left(z^{-1}\right)$ is the fitted admittance transfer function matrix which of the size $m \times m$ (where $m$ is the number of ports). By substituting $z=e^{i 2 \pi f T_{s}}$ in (9), the 
fitted admittance matrix data for $k$ frequency samples can be represented as

$$
\left[Y_{d}\right]_{m \times m \times k}=\left[Y_{f}\left(z^{-1}\right)\right]_{m \times m}
$$

where $f \in 1: f_{\max }$ ( $f_{\max }$ being the maximum frequency under consideration) and, $T_{s}$ is the sampling time. Let $G_{d}$ is the real part of the admittance matrix $\left(Y_{d}\right)$. Then

$$
\left[G_{d}\right]_{m \times m \times k}=\Re\left[Y_{d}\right]_{m \times m \times k}
$$

For a function to be passive

$$
\operatorname{eig}\left(G_{d}\right)>0
$$

This implies that, if the admittance transfer function matrix is positive definite then it is also passive. Considering this, if the fitted function $Y_{f}$ violates (32), then a new corrected transfer function matrix $Y_{c}$ is formulated. The conductance transfer function $\left(G_{f}\right)$ and susceptance transfer function $\left(B_{f}\right)$ are calculated as follows.

$$
\begin{gathered}
Y_{f}\left(z^{-1}\right)=G_{f}\left(z^{-1}\right)+B_{f}\left(z^{-1}\right) \\
G_{f}\left(z^{-1}\right)=\frac{1}{2}\left[Y_{f}\left(z^{-1}\right)+Y_{f}\left(z^{-1}\right)^{*}\right] \\
B_{f}\left(z^{-1}\right)=\frac{1}{2}\left[Y_{f}\left(z^{-1}\right)-Y_{f}\left(z^{-1}\right)^{*}\right]
\end{gathered}
$$

where $*$ stands for complex conjugate.

Since passivity is related to real part of admittance matrix $\left(G_{f}\right)$, correcting $G_{f}$ without affecting imaginary part $\left(B_{f}\right)$ is sufficient. Then $Y_{c}$ can be represented as follows

$$
Y_{c}\left(z^{-1}\right)=G_{c}\left(z^{-1}\right)+B_{f}\left(z^{-1}\right)
$$

where

$$
G_{c}\left(z^{-1}\right)=G_{f}\left(z^{-1}\right)+\Delta G
$$

The objective here is to calculate $\Delta G$. A real, symmetric matrix $G_{b}$ is said to be positive definite if $x^{T} G_{b} x>0 \forall$ $x \neq 0$. Thus $x^{T} G_{b} x$ can be written as

$$
x^{T} G_{b} x=\frac{1}{2} x^{T}\left(G_{b}+G_{b}^{T}\right) x
$$

This shows $G_{b}$ is positive definite if and only if $G_{b}+G_{b}^{T}$ is positive definite. This can be achieved by minimizing an objective function through optimization as

$$
\begin{aligned}
& \min \left\|G_{d}-G_{b}\right\|_{F} \\
& \text { s.t. } G_{b}+G_{b}^{T}>0
\end{aligned}
$$

where $F$ stands for Forbenius norm of a matrix. We propose a convex optimization formulation to find $G_{b}$ using semi definite programming (SDP) [23]. The optimization solution is then used to calculate $\Delta G$ using (41).

$$
\Delta G=G_{b}-G_{d}
$$

The validity of the proposed algorithm is implemented for enforcing passivity of 1-port network formulated previously. From Fig. 8 it can be seen that the passivity is enforced.

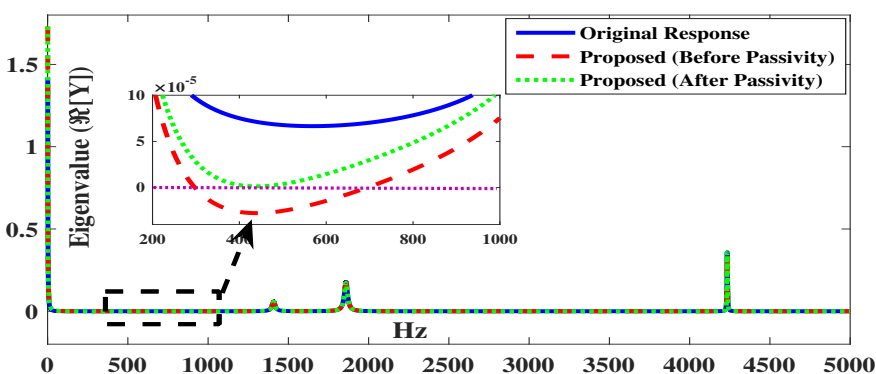

Fig. 8. The eigenvalue of real-part of admittance matrix (1-port)

3) Interfacing FDNE with real time simulator: FDNE can be directly implemented since it is computed in $z$-domain. The implementation process is as follows. With boundary bus voltage $\left(V_{b}\right)$ as input to FDNE, and with $n^{\text {th }}$ order estimation, (8) can be written as

$$
\begin{aligned}
I_{F}(k) & =-a_{1} I_{F}(k-1)-a_{2} I_{F}(k-2) \cdots-a_{n} I_{F}(k-n) \\
& +b_{1} V_{b}(k-1)+b_{2} V_{b}(k-2) \cdots+b_{n} V_{b}(k-n)
\end{aligned}
$$

where $I_{F}$ is current output from FDNE. For observing high frequency transients only FDNE part is required. To maintain boundary bus parameters at initial steady state, a constant current source is injected into the boundary bus as calculated from (43) [8], [9]. This can be represented as,

$$
\begin{gathered}
I_{b} \angle \delta_{b}=\left(\frac{P_{b}+j Q_{b}}{V_{b} \angle \theta_{b}}\right)^{*} \\
I_{b i n j} \angle \beta_{i n j}=I_{b} \angle \delta_{b}-Y_{c}(60 H z) V_{b} \angle \theta_{b}
\end{gathered}
$$

where $P_{b}$ and $Q_{b}$ are the active and reactive power flow respectively from the boundary bus, $V_{b}$ and $\theta_{b}$ are the voltage and angle respectively of the boundary bus. Since admittance at a fundamental component of frequency $\left(Y_{c}(60 \mathrm{~Hz})\right)$ is included either in (5) for EMT+FDNE+TSA Based Model or in (43) for EMT+FDNE Based Model, the fundamental frequency component must be eliminated from FDNE. This is performed by subtracting $Y(60 \mathrm{~Hz}) V_{b} \angle \theta_{b}$ term in (44) before injecting boundary bus current to remove fundamental frequency component from FDNE.

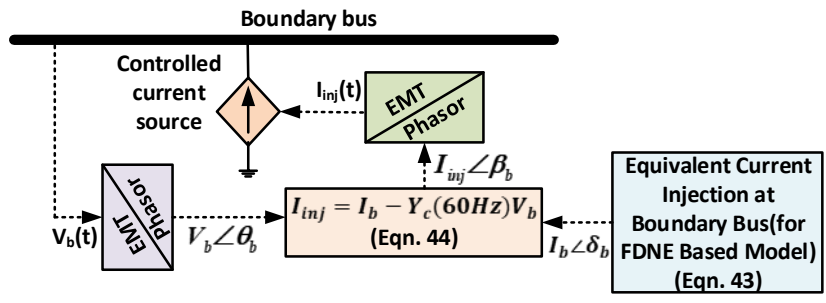

Fig. 9. Boundary bus current calculation for FDNE only

\section{Study and analysis of FDNE type equivalent modeling}

For study and preliminary analysis, the proposed FDNE algorithm is implemented on two area test system. Two cases are considered for study. First case is the proposed method EMT+FDNE Based Model (Proposed) and the second case is an existing architecture (EMT+FDNE Based Model $(V F))$. 


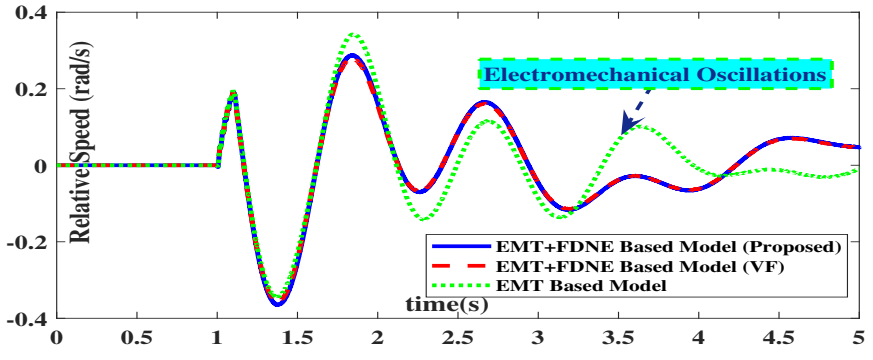

Fig. 10. Relative speed of Gen.2 w.r.t Gen.1

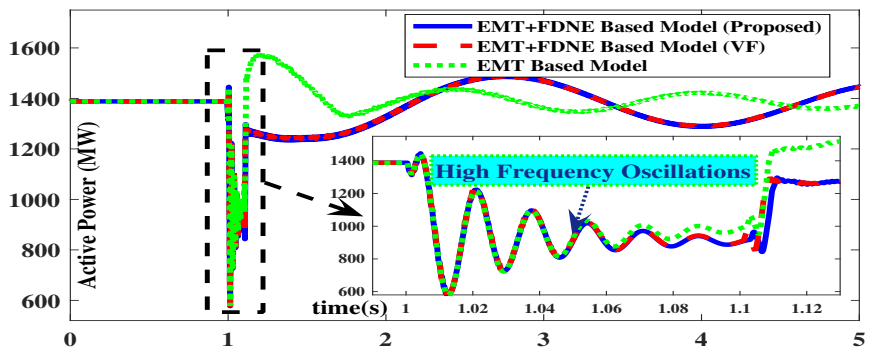

Fig. 11. Active power flow from bus 10 to bus 9 (Boundary Bus)

For validation, both the test cases are compared with EMT Based Model. For the same type of disturbance as discussed in Section II-B, Fig. 10 shows the comparison of the relative speed of Gen. 2 w.r.t gen. 1, and Fig. 11 shows the comparison of active power flow from bus 10 to bus 9 .

TABLE IV

COMPARISON OF REDUCED (EMT+FDNE) AND ORIGINAL (EMT) MODELS OF TWO-AREA SYSTEM

\begin{tabular}{ccc}
\hline & EMT+FDNE(Proposed) & EMT+FDNE(VF) \\
\hline Relative Speed (Fig. 10) & 0.481261 & 0.4827015 \\
\hline Active Power (Fig. 11) & 0.077046 & 0.0771309 \\
\hline
\end{tabular}

Table IV shows the comparisons between proposed FDNE and an offline VF based algorithm. Both algorithms gives similar results, proving that FDNE can be formulated online with less computational effort and lower order of transfer function when compared to offline algorithms. From Fig. 10 and Fig. 11 it can be seen that with this approach, high frequency oscillations are preserved whereas electromechanical oscillations are not preserved, proving the need to have combined FDNE models with TSA equivalents.

\section{E. Implementing TSA and FDNE on two area power system}

Fig. 12 shows the implementation approach for combined TSA and FDNE type equivalents. Table. $\mathrm{V}$ shows the comparison of frequency and damping factor for original model and various reduced order model using eigen value realization algorithm for generator-3 speed data. It can be seen that the proposed approach provides very close result compared to full EMT based model. Fig. 13 shows the comparison of the relative speed of Gen. 2 w.r.t gen. 1, and Fig. 14 shows the comparison of active power flow from bus 10 to bus 9. From Fig. 13 and Fig. 14, it can be seen that both high-frequency and electromechanical oscillations are well preserved. Table. VI shows the error comparison between orignial and reduced model using (7).

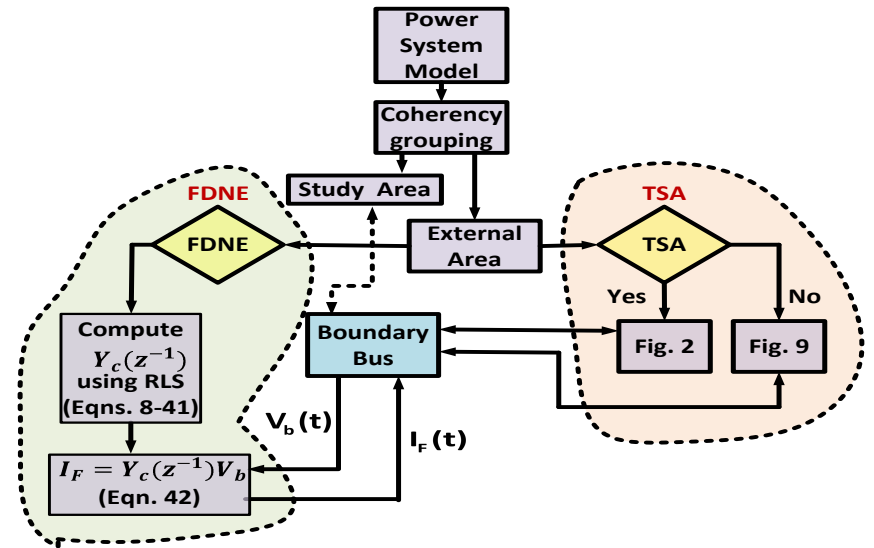

Fig. 12. Implementation flowchart for TSA and FDNE type models.

TABLE V

COMPARISON OF REDUCED ORDER (FDNE ONLY) MODELS

\begin{tabular}{lccc}
\hline Case & Eigen Value & Frequency(Hz) & Damping(\%) \\
\hline \multirow{2}{*}{ EMT Based Model } & $-1.323 \pm \mathrm{i} 7.544$ & 0.6566 & 21.92 \\
& $-0.880 \pm \mathrm{i} 5.157$ & 0.8327 & 16.82 \\
\hline EMT+TSA Based & $-0.906 \pm \mathrm{i} 7.096$ & 0.5494 & 43.09 \\
Model(AGG) & $-1.381 \pm \mathrm{i} 6.537$ & 1.0634 & 20.67 \\
\hline EMT+TSA Based & $-1.306 \pm \mathrm{i} 7.583$ & 0.6501 & 5.7543 \\
Model & $-0.598 \pm \mathrm{i} 5.014$ & 0.8038 & 11.8466 \\
\hline EMT+FDNE Based & $-0.664 \pm \mathrm{i} 6.993$ & 0.3822 & 61.26 \\
Model & $-0.373 \pm \mathrm{i} 2.507$ & 0.4034 & 61.26 \\
\hline EMT+FDNE+TSA & $-1.321 \pm \mathrm{i} 7.652$ & 0.6623 & 4.1457 \\
Based Model(AGG) & $-0.670 \pm \mathrm{i} 4.984$ & 0.8804 & 13.336 \\
\hline EMT+FDNE+TSA & $-1.325 \pm \mathrm{i} 7.601$ & 0.6518 & 5.2343 \\
Based Model & $-0.610 \pm \mathrm{i} 5.002$ & 0.8021 & 12.1216 \\
\hline
\end{tabular}

TABLE VI

COMPARISON OF REDUCED (EMT+FDNE+TSA) AND ORIGINAL (EMT) MODELS OF TWO-AREA SYSTEM

\begin{tabular}{ccc}
\hline & $\begin{array}{c}\text { EMT+FDNE+TSA } \\
\text { (AGG) }\end{array}$ & EMT+FDNE+TSA \\
\hline Relative Speed (Fig. 13) & 0.2286 & 0.0923 \\
\hline Active Power (Fig. 14) & 0.0163 & 0.0076 \\
\hline
\end{tabular}

\section{IMPLEMENTATION TEST ON INTERCONNECTED POWER GRID}

To prove scalability and implementation using a multi-port networks, IEEE 39 and 68 bus power system models are considered. Plesae refer [24] and [25] for IEEE 39 and 68 bus system details and one-line diagrams respectively.

\section{A. IEEE 39 Bus System Implementation Test Results}

In this system first, based on the coherency grouping of the generators the test system is divided into study and external area. To assess the performance of proposed $E M T+F D N E+T S A$ based reduced order model, Group-I which consists of generators 4, 5, 6, 7, 9 is considered as external area and the rest of the power system as study area. The study and external area is divided at bus 16 (Port-1), bus 17 (Port2), and bus 26 (Port-3). Fig. 15(a) and Fig. 15(b) shows the admittance magnitude and angle of port-3. Fig. 16 shows the passivity enforcement for 3-port network.

For analysis, a three-phase fault is initialized for a duration of $0.1 \mathrm{~s}$ and the simulation results are compared with the 


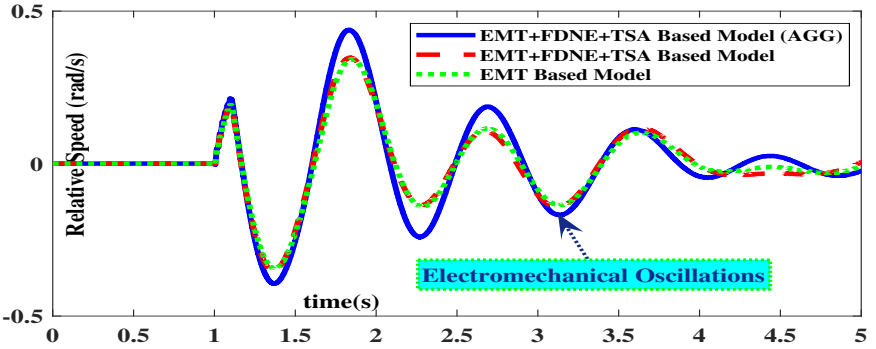

Fig. 13. Relative speed of Gen.2 w.r.t Gen.1

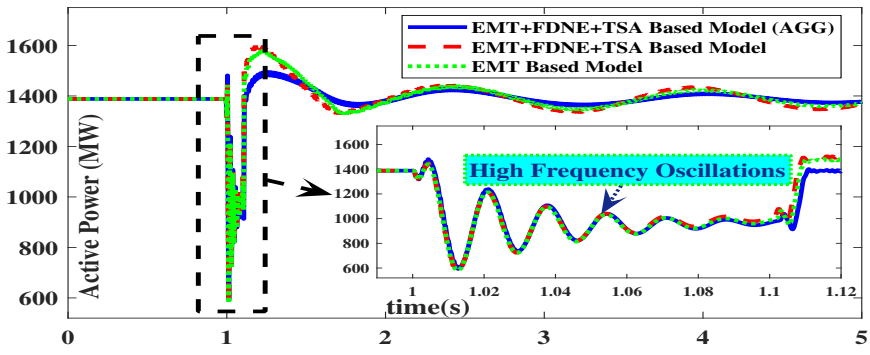

Fig. 14. Active power flow from bus 10 to bus 9 (Boundary Bus Power)

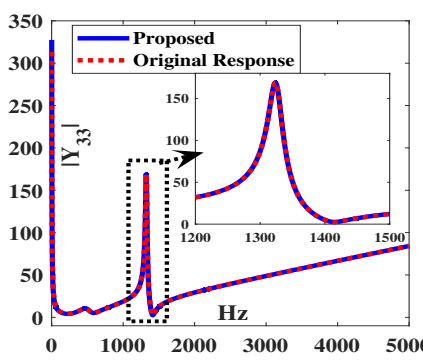

(a) Magnitude of admittance $\left(Y_{33}\right)$

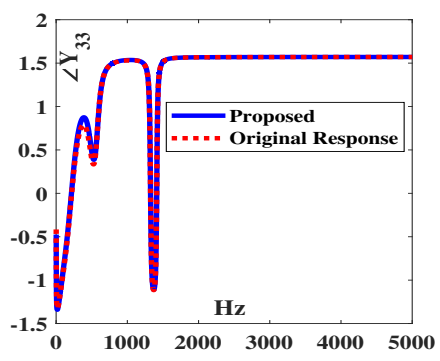

(b) Angle of admittance $\left(Y_{33}\right)$
Fig. 15. Admittance vs frequency of external area (39 Bus System)

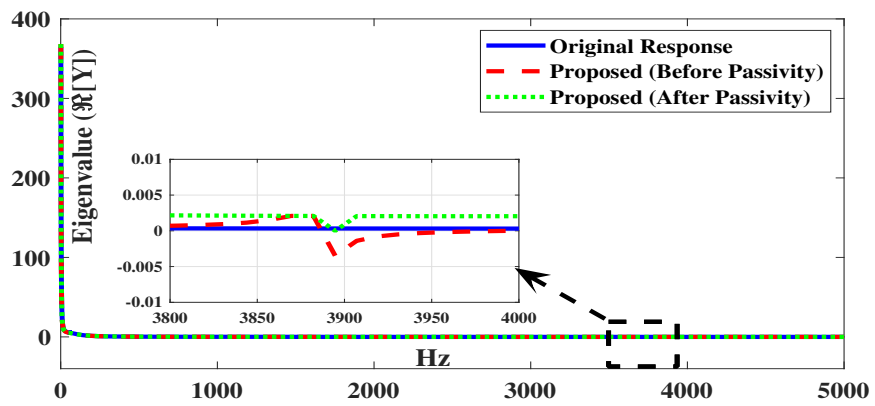

Fig. 16. The corrected eigenvalue of real-part of admittance matrix (3-port)

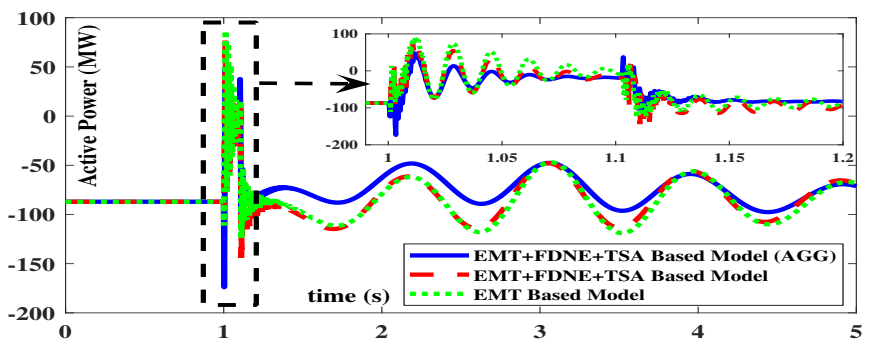

Fig. 17. Bus 26 Active Power

original model. Fig. 17 to Fig. 19 shows the validation results of the proposed algorithm. Table VII shows relative error (7) comparison of reduced and original models. It can be seen that

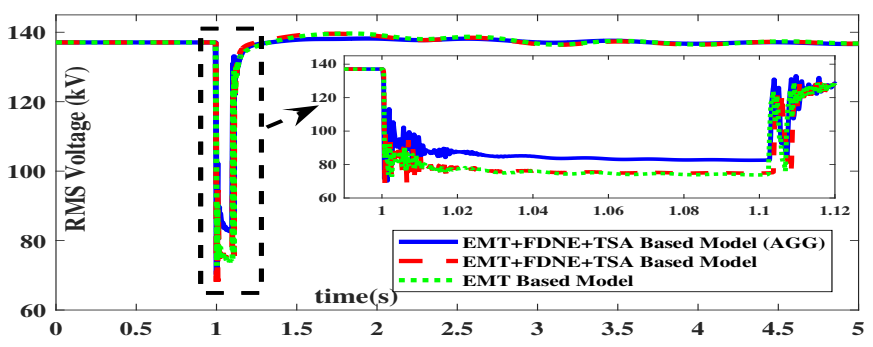

Fig. 18. Bus 17 Voltage

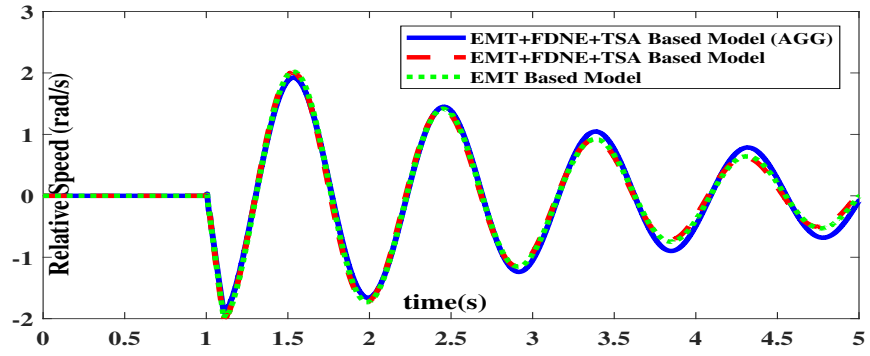

Fig. 19. Relative speed of generator gen.1 w.r.t gen.2

TABLE VII

COMPARISON OF REDUCED (EMT+FDNE+TSA) AND ORIGINAL (EMT) MODELS OF IEEE 39 BUS SYSTEM

\begin{tabular}{ccc}
\hline & $\begin{array}{c}\text { EMT+FDNE+TSA } \\
\text { (AGG) }\end{array}$ & EMT+FDNE+TSA \\
\hline Active Power (Fig. 17) & 0.1634 & 0.0489 \\
\hline Voltage (Fig. 18) & 0.0112 & 0.0081 \\
\hline Relative Speed (Fig. 19) & 0.0905 & 0.0433 \\
\hline
\end{tabular}

the reduced model closely resembles the full EMT model.

\section{B. IEEE 68 Bus System Implementation Test Results}

Further, in order to validate the proposed algorithm on a larger system, IEEE 68 bus system which consists of 16 generators consists of 5 -areas is considered. The area with generators 1 to 9 is considered as external area. The study and external areas are divided at bus 54 (Port-2), 27 (Port-3), and 60 (Port-1). Fig. 20(a) and Fig. 20(b) shows the admittance magnitude and angle of port-2. For analysis a 3-ph fault for a duration of $0.2 \mathrm{~s}$ is simulated at bus- 49 starting at at $1 \mathrm{~s}$. The responses of reduced order model are compared with the original model. Fig. 21 shows the Bus-60 voltage and Fig. 22 shows the relative speed of generator-10 w.r.t generator- 16 .

From the above comparisons it can be seen that the dynamics of the proposed reduced order model is similar to that of the original model. The computational time required to generate equivalents is shown in Table. VIII.

TABLE VIII

TIME REQUIRED FOR GENERATION OF EQUIVALENTS

\begin{tabular}{cccc}
\hline Model & TSA(AGG) & TSA & FDNE \\
\hline Two-Area System & $10 \mathrm{~s}$ & $8 \mathrm{~s}$ & $40 \mathrm{~s}$ \\
\hline 39 Bus System & $14 \mathrm{~s}$ & $13 \mathrm{~s}$ & $91 \mathrm{~s}$ \\
\hline 68 Bus System & $19 \mathrm{~s}$ & $17 \mathrm{~s}$ & $97 \mathrm{~s}$ \\
\hline
\end{tabular}

IV. CONCLUSION

In this paper a novel real-time frequency based reduced order modeling of the large power system for EMT simulation 


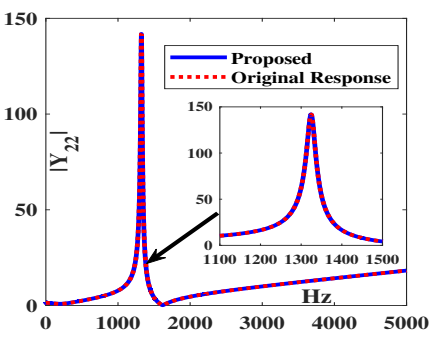

(a) Magnitude of admittance $\left(Y_{22}\right)$

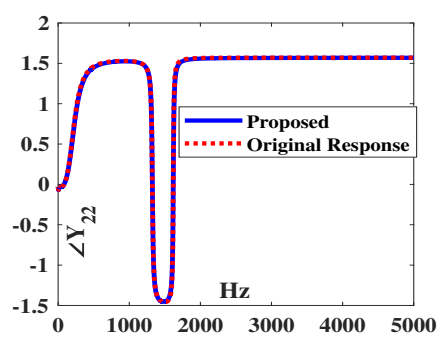

(b) Angle of admittance $\left(Y_{22}\right)$
Fig. 20. Admittance vs frequency of external area (68 Bus System)

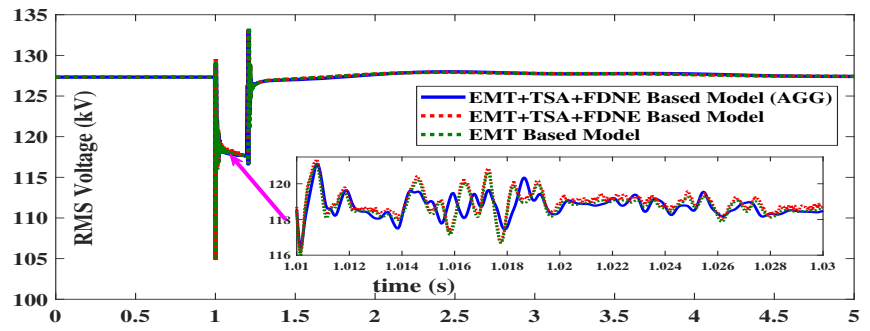

Fig. 21. Bus 60 Voltage

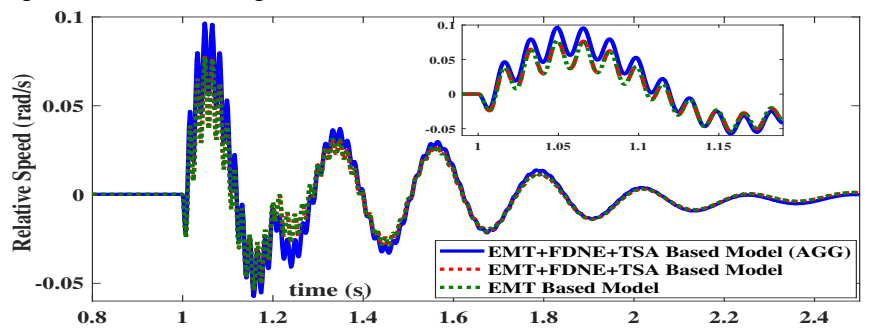

Fig. 22. Relative speed of generator 10 w.r.t generator 16

is proposed. In the proposed architecture, external area is modeled as a combination of FDNE and TSA. FDNE is formulated using an online discrete RLS which can preserve high-frequency behavior, where as TSA preservers the electromechanical (low frequency) behavior of the system under consideration. The approach also enforces passivity conditions. Implementation results in two area, IEEE 39 and 68 bus power system models shows that the proposed reduced order model represents the aggregated power grid accurately and at the same time can capture the oscillations as close as the full detail model for EMT simulations.

\section{REFERENCES}

[1] P. Sorensen and A. D. Hansen and P. Christensen and M. Mieritz and J. Bech and B. Bak-Jensen and H. Nielsen, Simulation and verification of transient events in large wind power installations. Ris National Laboratory, Rosklide, 2003.

[2] S. Abourida and J. Belanger and V. J. Marandi, Real-Time Power System Simulation: EMT vs. Phasor. OPAL-RT Technologies: White Paper: opWP150620-sa-revA, 2016.

[3] A. Ubolli and B. Gustavsen, "Multiport frequency-dependent network equivalencing based on simulated time-domain responses," IEEE Trans. on Power Del., vol. 27, no. 2, pp. 648-657, Apr 2012.

[4] J. H. Chow, Power system coherency and model reduction. New York, NY, USA: Springer, 2013.

[5] X. Guillaud et al., "Applications of real-time simulation technologies in power and energy systems," IEEE Power Energy Technol. Syst. J., vol. 2, no. 3, pp. 103-115, Sep 2015.

[6] B. Gustavsen and C. Heitz, "Fast realization of the modal vector fitting method for rational modeling with an accurate representation of small eigenvalues," IEEE Trans. on Power Del., vol. 24, no. 3, pp. 1396-1405, Jul 2009.
[7] B. Gustavsen, "Rational modeling of multiport systems via a symmetry and passivity preserving mode-revealing transformation," IEEE Trans. on Power Del., vol. 29, no. 1, pp. 199-206, Feb 2014.

[8] X. Lin, A. M. Gole, and M. Yu, "A wide-band multi-port system equivalent for real-time digital power system simulators," IEEE Trans. on Power Syst., vol. 24, no. 1, pp. 237-249, Feb 2009.

[9] Y. Liang, X. Lin, A. M. Gole, and M. Yu, "Improved coherency-based wide-band equivalents for real-time digital simulators," IEEE Trans. on Power Syst., vol. 26, no. 3, pp. 1410-1417, Jul 2011.

[10] Y. Zhang, A. M. Gole, W. Wu, B. Zhang, and H. Sun, "Development and analysis of applicability of a hybrid transient simulation platform combining TSA and EMT elements," IEEE Trans. on Power Syst., vol. 28, no. 1, pp. 357-366, Feb 2013.

[11] A. Ubolli and B. Gustavsen, "Comparison of methods for rational approximation of simulated time-domain responses: ARMA, ZD-VF, and TD-VF," IEEE Trans. on Power Del., vol. 26, no. 1, pp. 279-288, Jan 2011.

[12] K. Sheshyekani and B. Tabei, "Multiport frequency-dependent network equivalent using a modified matrix pencil method," IEEE Trans. on Power Del., vol. 29, no. 5, pp. 2340-2348, Oct 2014.

[13] X. Zhang, A. J. Flueck, and S. Abhyankar, "Implicitly coupled electromechanical and electromagnetic transient analysis using a frequencydependent network equivalent," IEEE Trans. on Power Del., vol. 32, no. 3, pp. 1262-1269, Jun 2017.

[14] A. Thakallapelli, S. Ghosh, and S. Kamalasadan, "Real-time reduced order model based adaptive pitch controller for grid connected wind turbines," in Proc. Industry Applications Society Annual Meeting (IAS), IEEE, Oct 2016, pp. 1-8.

[15] S. Ghosh and N. Senroy, "The localness of electromechanical oscillations in power systems," Int. J. Elect. Power Energy Syst., vol. 42, no. 1, pp. 306-313, Nov 2012.

[16] Power system analysis. [Online]. Available: http://nptel.ac.in/courses/ Webcourse-contents/IIT-Kanpur/power-system/chapter_3/3_6.html

[17] A. Thakallapelli, S. J. Hossain, and S. Kamalasadan, "Coherency and online signal selection based wide area control of wind integrated power grid," IEEE Trans. Industrial Applications, vol. 54, no. 4, pp. 37123722, Mar 2018.

[18] J. Chow, P. Accari, and W. Price, "Inertial and slow coherency aggregation algorithms for power system dynamic model reduction," IEEE Trans. on Power Syst., vol. 10, no. 2, pp. 680-685, May 1995.

[19] Z. Stojkovic , Computer aided design in power engineering-application of software tools. Springer, 2012.

[20] A. Thakallapelli, S. Ghosh, and S. Kamalasadan, "Real-time frequency based reduced order modeling of large power grid," in Proc. Power and Energy Society General Meeting, IEEE, Jul 2016, pp. 1-5.

[21] A. Thakallapelli, S. J. Hossain, and S. Kamalasadan, "Coherency based online wide area control of renewable energy integrated power grid," in Proc. PEDES, IEEE, Dec 2016, pp. 1-6.

[22] K. J. Astrom and B. Wittenmark, Adaptive control. Addison-Wesley Publishing Company, 1995.

[23] M. Grant and S. Boyd, "Graph implementations for nonsmooth convex programs," in in Recent Advances in Learning and Control, New York: Springer, 2008, pp. 95-110.

[24] I. Hiskens, "Report: 39-bus system (new england reduced model)," IEEE PES Task Force on Benchmark Syst. for Stability Controls, 2013.

[25] A. K. Singh and B. C. Pal, Report on the 68-bus, 16-machine, 5-area system. IEEE PES Task Force on Benchmark Systems for Stability Controls, Ver. 3.3, 2013.

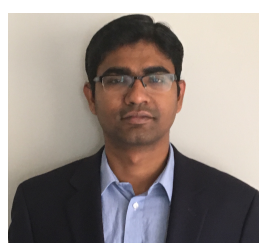

A. Thakallapelli (S'14) received his B.Tech degree in Electrical Engineering from Acharya Nagarjuna University in 2010 and the M.Tech degree in Electrical Engineering from the Veermata Jijabai Technological Institute in 2012. He is currently working toward the Ph.D degree in Electrical Engineering from the Department of Electrical and Computer Engineering, University of North Carolina at Charlotte. His research interests include wide-area control, reduced order modeling, power system stability and renewable energy. 


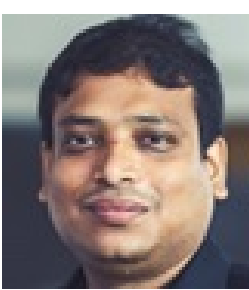

S. Ghosh (M'12) received the B.SC Engg.from National Institute of Technology (NIT) Jamshedpur, India, in 2002, the M.Tech degree from NIT Durgapur, India, in 2009, and the Ph.D. degree from Indian Institute of Technology, Delhi, India, in 2013. Currently, he is working in Khalifa University, Abu Dhabi. He worked as an Associated Graduate Faculty member with the Department of Electrical and Computer Engineering, University of North Carolina, Charlotte, NC, USA and as an Assistant Professor with the Department of Electrical Engineering, Indian School of Mines, Dhanbad, India. His research interests include power system stability, model order reduction, and grid integration with renewable energy resources.

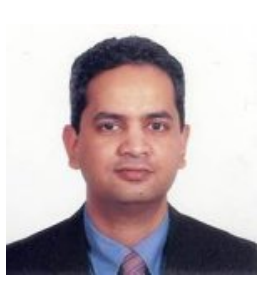

S. Kamalasadan (S'01, M'05, SM'17) received his B Tech. degree in Electrical and Electronics from the University of Calicut, Kerala, India in 1991, M.Eng in Electrical Power Systems Management, from the Asian Institute of Technology, Bangkok, Thailand in 1999, and Ph.D. in Electrical Engineering from the University of Toledo, Ohio, USA in 2004. He is currently working as a Professor in the department of electrical and computer engineering at the University of North Carolina at Charlotte. He has won several awards including the NSF CAREER award and IEEE best paper award. His research interests include Intelligent and Autonomous Control, Power Systems dynamics, Stability and Control, Smart Grid, Micro-Grid and Real-time Optimization and Control of Power System. 